\title{
RADIOACTIVE POLLUTION ESTIMATE FOR FUKUSHIMA NUCLEAR POWER PLANT BY A PARTICLE MODEL
}

\author{
Keisuke Saito ${ }^{\mathrm{a}, *}$ and Susumu Ogawa ${ }^{\mathrm{b}}$ \\ ${ }^{a}$ Nagasaki University, Graduate school of engineering, Bunkyo-machi, Nagasaki, 852-8521 Japan - \\ jj20140051@cc.nagasaki-u.ac.jp \\ ${ }^{\mathrm{b}}$ Nagasaki University, Graduate school of engineering, Bunkyo-machi, Nagasaki, 852-8521 Japan - \\ ogawasusumu@nagasaki-u.ac.jp
}

Commission VIII, WG VIII/1

KEY WORDS: Fukushima, Radioactive pollution, Particle model,

\begin{abstract}
:
On Mar 12, 2011, very wide radioactive pollution occurred by a hydrogen explosion in Fukushima Nuclear Power Plant. A large amount of radioisotopes started with four times of explosions. With traditional atmospheric diffusion models could not reconstruct radioactive pollution in Fukushima. Then, with a particle model, this accident was reconstructed from meteorological archive and Radar- AMeDAS. Calculations with the particle model were carried out for Mar 12, 15, 18 and 20 when east southeast winds blew for five hours continuously. Meteorological archive is expressed by wind speeds and directions in five-km grid every hour with eight classes of height till $3000 \mathrm{~m}$. Radar- AMeDAS is precipitation data in one-km grid every thirty minutes. Particles are ten scales of 0.01 to $0.1 \mathrm{~mm}$ in diameter with specific weight of 2.65 and vertical speeds given by Stokes equation. But, on Mar 15, it rained from 16:30 and then the particles fell down at a moment as wet deposit in calculation. On the other hand, the altitudes on the ground we re given by DEM with $1 \mathrm{~km}$-grid. The spatial dose by emitted radioisotopes was referred to the observation data at monitoring posts of Tokyo Electric Power Company. The falling points of radioisotopes were expressed on the map using the particle model. As a result, the same distributions were obtained as the surface spatial dose of radioisotopes in aero-monitoring by Ministry of Education, Culture, Sports, Science and Technology. Especially, on Mar 15, the simulated pollution fitted to the observation, which extended to the north west of Fukushima Daiichi Nuclear Power Plant and caused mainly sever pollution. By the particle model, the falling positions on the ground were estimated each particle size. Particles with more than $0.05 \mathrm{~mm}$ of size were affected by the topography and blocked by the mountains with the altitudes of more than $700 \mathrm{~m}$. The particle model does not include the atmospheric stability, the source height, and deposit speeds. The present assignment is how to express the difference of deposition each nucleus.
\end{abstract}

\section{INTRODUCTION}

On March 11, 2011, the Great East Japan Earthquake brought the earthquake and tsunami in East Japan, and Fukushima Daiichi Nuclear Power Plant became out of control, finally worldwide radioisotope pollution occurred. Especially hydrogen explosions from March 12 to 15 and attendant vent operation emitted radioisotopes and impacted neighboring areas significantly. Atmospheric diffusion of radioisotopes was simulated by SPEEDI and various methods so far as validations and reproduction (Japan Atomic Energy Agency, 2011). But we had few data at that time and the phenomena was complex, therefore, depending on methods and used parameters, consistency between monitoring results and other validation methods had some gap and uncertainty of calculation results still remain very much (Ohara et al., 2012). Although in 2012 Tokyo Electric Power Company presented analytical results on radioisotope emission estimation (Tokyo Electric Power Company, 2012), still the estimate at the accident and the examination for simulation methods are modified continuously.

In this study, an atmospheric diffusion simulation was carried out using a simple particle model. In this method, most radioisotopes were assumed to move in hydrogen explosions with concrete debris or aerosol, and their flying paths and falling points were estimated from meteorological archive and DEM to validate their diffusion states. Without complex and vast calculation, instantaneous diffusion damage predictions in the accident or trial atmospheric diffusion simulation before the accident are expected with much effect.

In this paper, using the above method radioisotope diffusion was examined especially from March 12 to 20 when most radioisotopes were emitted.

\subsection{Outline of the Fukushima nuclear power plant accident}

At 14:46 on March 11, 2011, the great earthquake with magnitude of 9.0 occurred from a focus of Sanriku-oki. At 15:50, the great tsunami attacked Fukushima Daiichi and Daini Nuclear Power Plants. The emergent core cooling system did not work and lost cooling ability. Fukushima Daiichi had meltdown at Reactors 1 to 3, and a hydrogen explosion occurred, which made building structures flying to pieces and a big amount of isotopes leaking into the atmosphere. At 13:10 on March 20, Reactor 4 was fired. On March 21, it rained in the Kanto plain, and high concentration of iodine 131 was detected from drinking water in waterworks in the Tone river watershed on March 22 and 23.

\subsection{Location}

Fukushima nuclear power plant is located in the place $280 \mathrm{~km}$ away from Tokyo. On the east side it faces the Pacific Ocean, from which tsunami flowed in. On the other hand, on the west

\footnotetext{
* Corresponding author
} 
side it faces Abukuma highlands, where the flat land near the plant continues to steep slopes on the surroundings. Location of Fukushima nuclear power plant is shown in Figure 1.

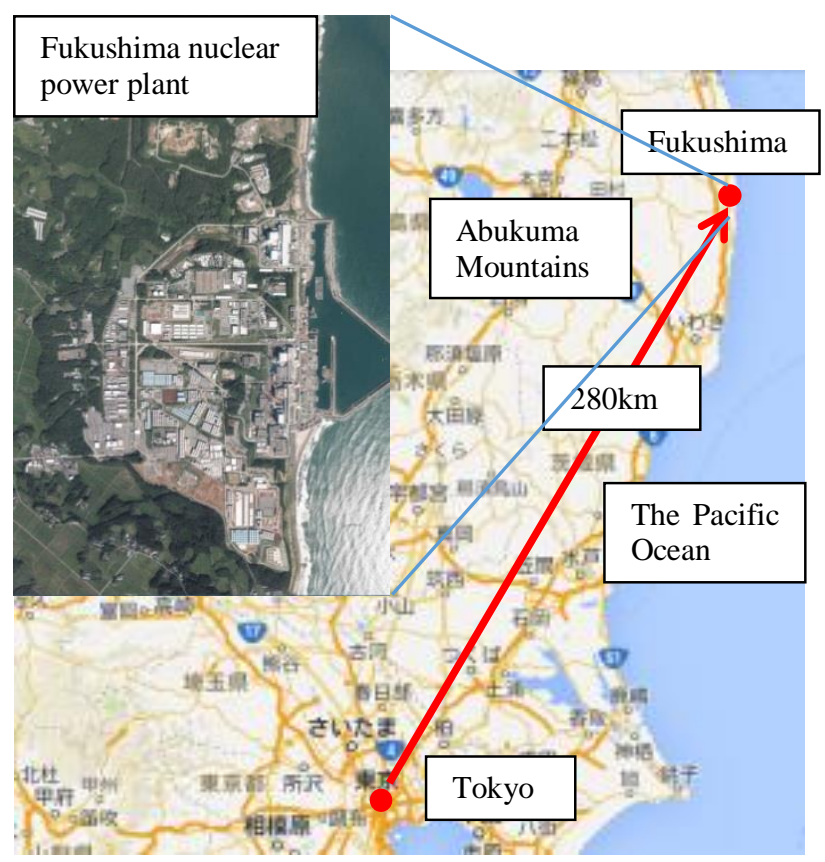

Figure 1. Location of Fukushima nuclear power plant

\section{METHODS}

\subsection{Outlines}

In advection diffusion of radioisotopes in the air, mostly an atmospheric diffusion model and a particle model are available. The atmospheric diffusion model is often used for process of migration for gaseous contamination, but various parameters are required: atmospheric stability, the source height, a mixing layer height, and a deposition rate. On the other hand, in a particle model, since only particle sizes are a parameter for calculation, the total calculation is very simple as characteristics. In this study, most radioisotopes were emitted from Fukushima Daiichi Nuclear Power Plant by hydrogen explosions, vent operations, and leak with pressure increase in the containment vessels, and they are assumed to move with concrete debris in hydrogen explosions or aerosols in the air using a particle model.

\subsection{Used data}

2.2.1 Wind directions and velocities: In this study, wind velocity and direction data were from hourly atmospheric analytical data, GPV (Grid Point Value) in the mesoscale model, MSM. Hourly atmospheric analytical data, GPV are modified by the wind profiler observation from wind predicted data in MSM as the initial data. These data are analytical ones of surface winds and winds aloft (Meteorological Bureau, 2006). If the wind profiler observation such as AMeDAS is available, this value and its distance correct the values in MSM and accurate wind direction and velocity data are offered. Since the format is constant without wind profiler observation, the uniform continuous calculation is always possible. The spatial resolution is 0.05 deg. by $0.0625 \mathrm{deg}$. and 16 layers in elevation for surface winds and winds-aloft.

2.2.2 Precipitation: In this study, precipitation data were used from the composite data of RADAR and AMeDAS in
Meteorological Bureau. The composite data of RADAR and AMeDAS in Meteorological Bureau are obtained from radars and AMeDAS, areal rain gages in the surface in Japan with $1-\mathrm{km}$ grids. The data are shown in hourly precipitation every 30 minutes (Meteorological Bureau). Since the format is constant without areal data, the uniform continuous calculation is always possible even if the nearest areal observation data is not available by the accident in this study. In this study these data were used for the judgement dry deposit and wet deposit.

2.2.3 Topography data: In this study, the third grid data, elevations and slopes in the digital national land information were used. These data spatial resolution is $1 \mathrm{~km}$ by $1 \mathrm{~km}$. The elevation maps open in Japan have fine grid data, but $1 \mathrm{~km}$ by 1 $\mathrm{km}$ grids were used in this study corresponding to wind direction and velocity data and precipitation data with $1 \mathrm{~km}$ by $1 \mathrm{~km}$ grids and the temporal resolution with 10 minutes. These data include the mean, maximum, minimum and elevations, but the mean data were used.

Used data for the analysis were as Table 1.

\begin{tabular}{|l|l|c|}
\hline \multicolumn{1}{|c|}{ Data } & \multicolumn{1}{|c|}{ Data name } & Spatial resolution \\
\hline $\begin{array}{l}\text { Wind } \\
\text { data }\end{array}$ & $\begin{array}{l}\text { Hourly atmospheric } \\
\text { analysis data, GPV* }\end{array}$ & $\begin{array}{c}0.05 \mathrm{deg} . \times \\
0.0625 \mathrm{deg} .\end{array}$ \\
\hline $\begin{array}{l}\text { Precipit } \\
\text { ation }\end{array}$ & Radar -AMEDAS & $1 \mathrm{~km} \times 1 \mathrm{~km}$ \\
\hline DEM & $\begin{array}{l}\text { Digital national land } \\
\text { information, altitude and } \\
\text { slopes, 3D grid data }\end{array}$ & $1 \mathrm{~km} \times 1 \mathrm{~km}$ \\
\hline
\end{tabular}

* Meteorological Bureau (2006),

** Meteorological Bureau,

*** Ministry of Land

Table 1. Used data for calculation

\subsection{Objective area}

Objective area is shown in Figure 2, which is the most polluted from Aerial monitoring by Ministry of Education, Culture, Sports, Science and Technology (2011). In the square 50km inland and 50km north from Fukushima Daiichi Nuclear Power Plant, $1 \mathrm{~km}$ by $1 \mathrm{~km}$ grids were calculated as shown in Figure 2.

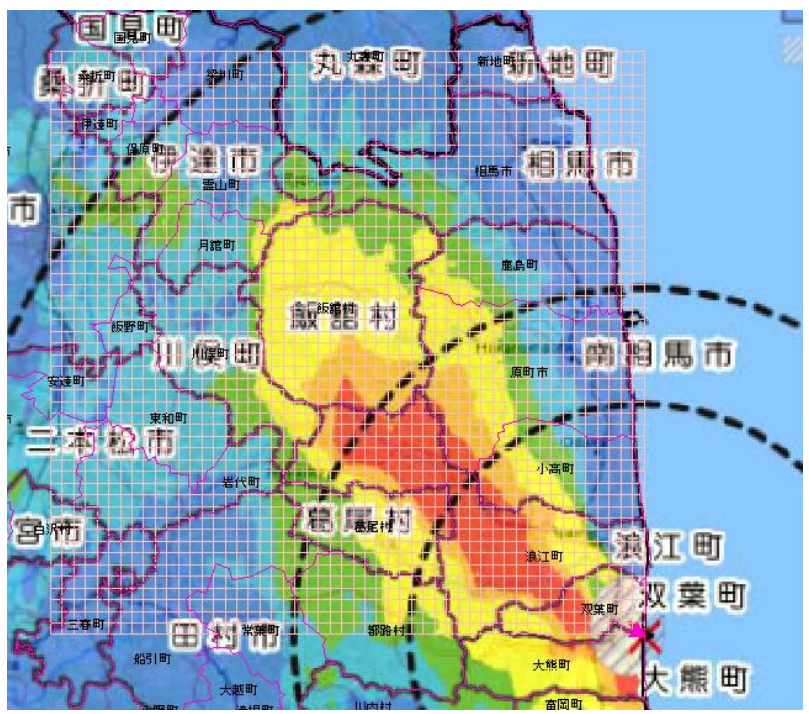

Figure 2. Aerial monitoring results and the objective area in this study 


\subsection{Calculation methods}

As described above, in this calculation, polluted radioisotopes were treated as a particle. Originally, radioisotopes were gaseous at leaked parts in the nuclear power plant, but actually most radioisotopes adhered on the concrete debris in hydrogen explosions and aerosols in the air and crystallized, finally travelled in the air and fell down on the ground. In this study, this state was assumed as a particle with a diameter of 0.01 to $0.1 \mathrm{~mm}$ in the calculation. Polluted particles were two types: in a gaseous state in a constant elevation speed and flying state in hydrogen explosions. Here, all particles started at 16 layers in elevation in GPV, hourly atmospheric analytical data and travelled with winds and constant falling velocities as shown in Figure 3.

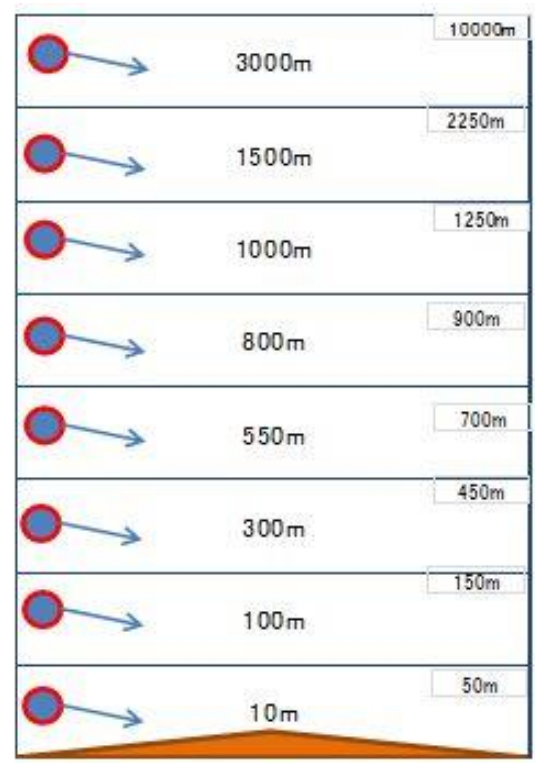

Figure 3. Box model of winds for the particle model

Horizontal velocities are the same as wind data, while vertical velocities are Stokes equation as next.

$$
v_{s}=\frac{D^{2}\left(\rho_{p}-\rho_{a}\right)}{18 \mu}
$$

$$
\begin{aligned}
& \text { where } \quad \begin{array}{l}
v_{s}: \text { a falling velocity }(\mathrm{m}), \\
\rho_{p}: \text { particle density }\left(2650 \mathrm{~kg} / \mathrm{m}^{3}\right) \\
\rho_{a}: \text { air density }\left(1.225 \mathrm{~kg} / \mathrm{m}^{3}\right) \\
\mu: \text { viscosity coefficient }\left(1.8 \cdot 10^{-5} \mathrm{Pas}\right) .
\end{array}
\end{aligned}
$$

The judgement for deposit used elevation values. From this, over Abukuma Hills the advective flow state of radioisotopes was examined topographically. Each calculation was ended when each particle fell down and flied out of the calculating area.

\section{RESULTS}

\subsection{Particle model}

Main explosions occurred four times (Table 2). But, only reactor 4 explosion increased spatial dose, while other explosions did not increase spatial dose. Moreover, in reactor 4 explosion, wind direction was north.

\begin{tabular}{|l|l|l|l|}
\hline Reactor 1 & Reactor 2* & Reactor 3 & Reactor 4 \\
\hline Mar12 & Mar15 & Mar14 & Mar15 \\
15:36 & $18: 10$ & $11: 01$ & $6: 14$ \\
\hline SE & SE & W & N \\
\hline
\end{tabular}

*Reactor 2 exploded without hydrogen.

Table 2. Hydrogen explosions and wind directions

From this fact, the pollution northwest of Fukushima Daiichi Nuclear Power Plant might be contributed by explosions March 12 and 15, Reactor 1 and 2, respectively. Thus, the objective calculation was started from the time when hydrogen explosions occurred on March 12 and 15. By a particle model, pollution state on March 12 was estimated as Figure 4. Circles expressed particle sizes. At this time wind directions were different very much each altitude: small particles trended to flow to the Pacific Ocean, while large particles fell down to the northwest from Fukushima Daiichi Nuclear Power Plant. From this result, particles emitted by an explosion on March 12 did not seem to approach west of Abukuma Hills by the effects of winds and geography. Over $3000 \mathrm{~m}$ by the effect of westerlies particles were transported to the Pacific Ocean and did not affect inland pollution.

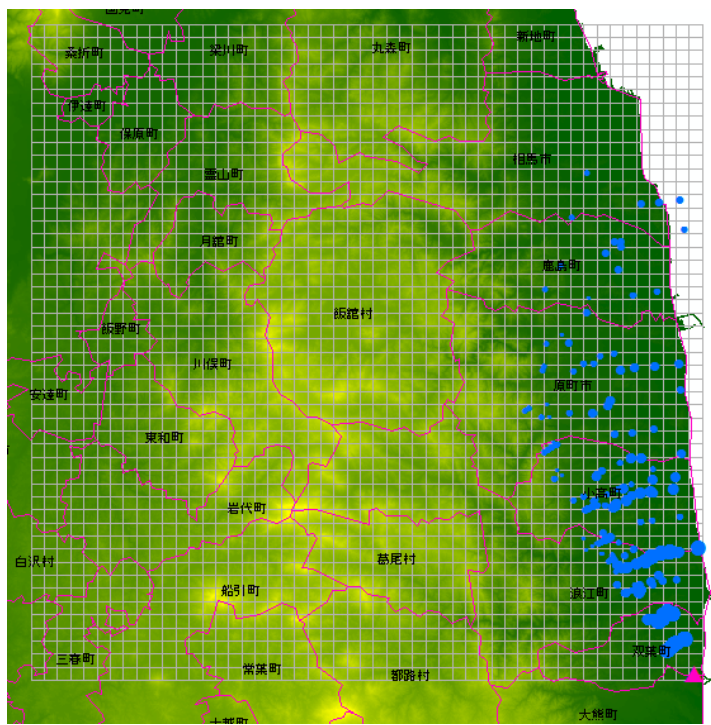

Figure 4. Particle trajectory estimated from wind directions and velocities on March 12

On the other hand, at 16:00 on March 15 it was rainy northwest of Fukushima Daiichi Nuclear Power Plant (Figure 5). At 18:10, Reactor 2 exploded with hydrogen. Rain clouds passed over Fukushima city from west, and at 21:00 they caught pollution particles east of Fukushima Prefecture (Figure 6). In this calculation, particles that entered rain grids fell down on the ground, but they continued to travel in the rain clouds. Wet deposit continued on the ground through the trajectory of particles. As a result, radioisotopes flied over Abukuma Hills and approached the west slopes of mountains. Till 24:00 most particles deposited wetly (Figure 7). Here, the backgrounds in Figures 6 and 7 used meteorological archive data (National Institute of Informatics) around Fukushima Daiichi Nuclear Power Plant. Particles lower than height $800 \mathrm{~m}$ were estimated to deposit wetly. Particles over $1000 \mathrm{~m}$ passed to the Pacific Ocean in the same as March 12. The calculated results are shown in Figure 8. 


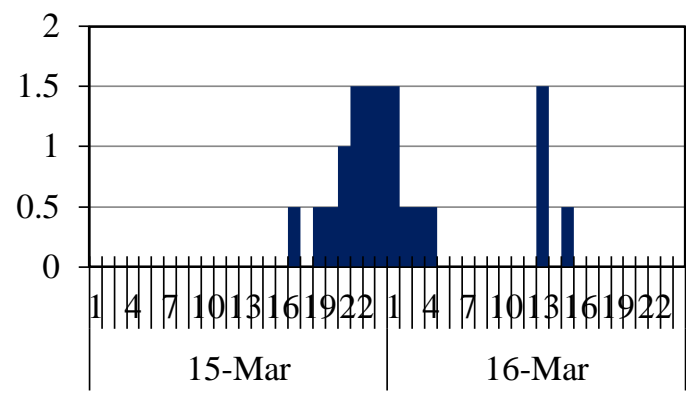

Figure 5. Hyetograph on March 15 to 16 at objective area

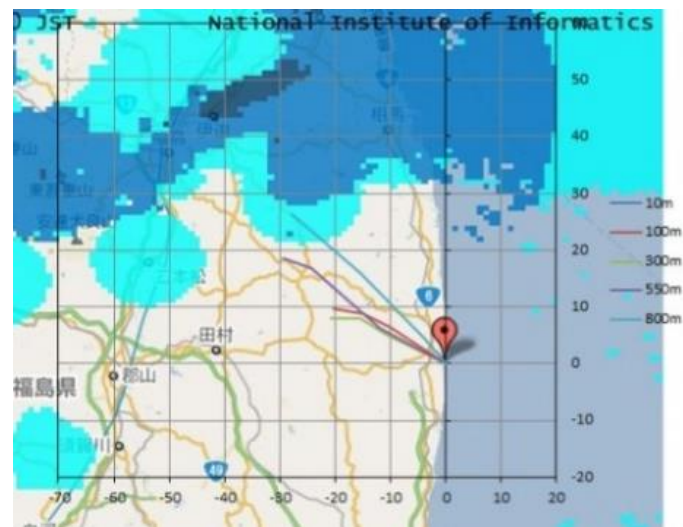

Figure 6. Flying particles and rain clouds at 21:00

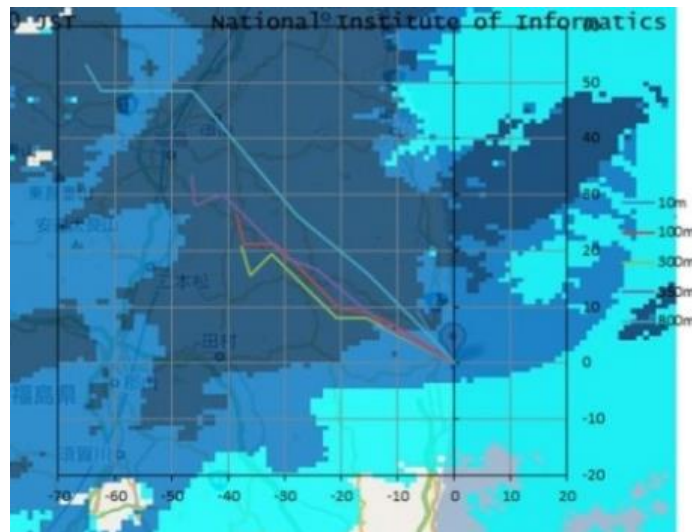

Figure 7. Flying particles and rain clouds at 0:00

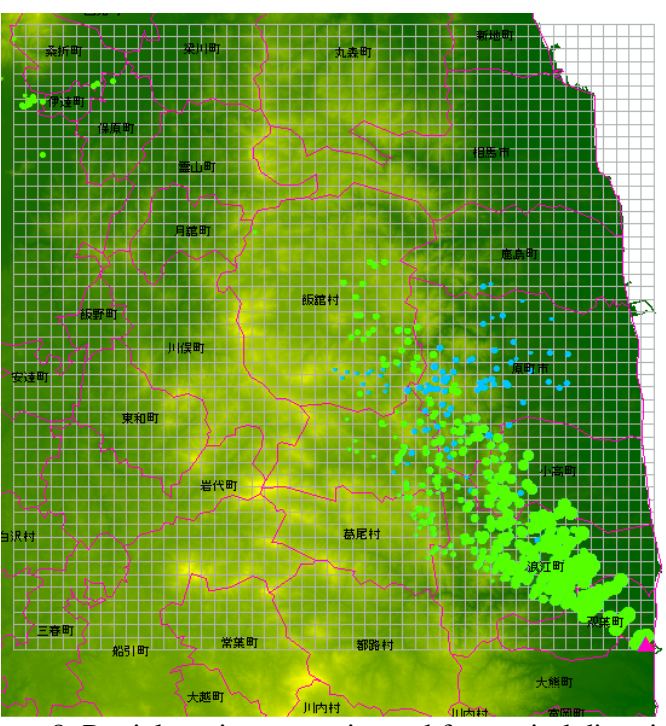

Figure 8. Particle trajectory estimated from wind directions and velocities on March 15.

Green circles: dry deposit, Blue circles: wet deposit.

Moreover, from the observation of radioisotopes at monitoring posts of Tokyo Electric Power Company (Tokyo Electric Power Company), a time series of March 12 to 21 with intense spatial dose and pollution time on northwest of Fukushima Daiichi Nuclear Power Plant with east to south winds were summarized and the results are shown in Figure 9. The bar graph shows the spatial dose each time and red bars indicate the time when east to south winds blew. Then, the spatial dose was very high on March 18 and 20 by east to south winds. Therefore, using a particle model, advection calculation was carried out.

The result on March 18 is shown in Figure 10. On this day, most of particles flied to northwest, but did not fly over the Abukuma Hills and concentrated within $15 \mathrm{~km}$ from the nuclear power plant. Less than $0.02 \mathrm{~mm}$ particles trended to fly to north.

Next, the result on March 20 is shown in Figure 11. Most of particles on this day trended to fly to north northwest uniformly in height and particle sizes. The horizontal distribution of polluted particles was more widely than other three days.

Figure 12 shows the total advection calculation results of radioisotopes on March 12, 15, 18, and 20 overlapped with aircraft monitoring results. In aircraft monitoring results, the most polluted areas fitted very well with flying traces on March 15 and 18. From this result, the pollution on northwest of Fukushima Daiichi nuclear power plant was estimated by the leak of radioisotopes and their movement.

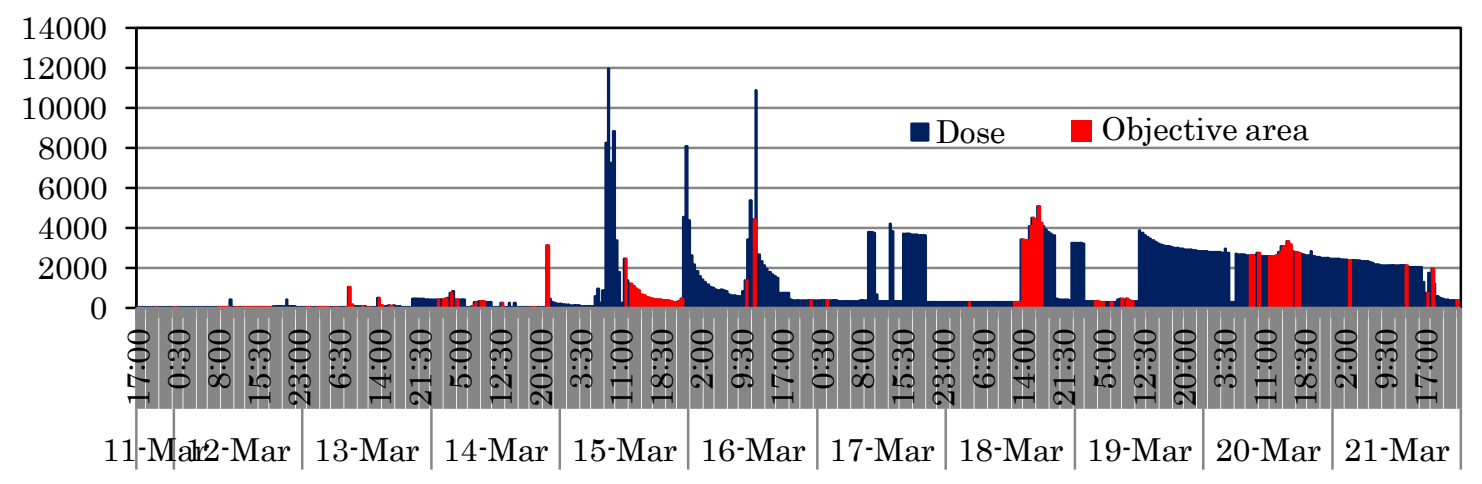

Figure 9. A time series of spatial dose (unit: $\mu \mathrm{Sv} / \mathrm{h}$ ) and the ratio of pollution for Objective area. 


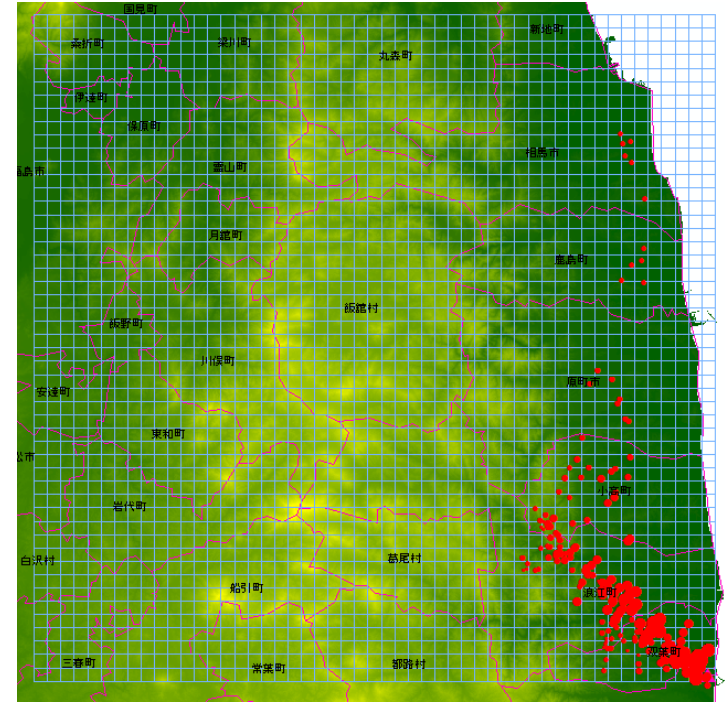

Figure 10. Particle trajectory estimated from wind directions and velocities on March 18

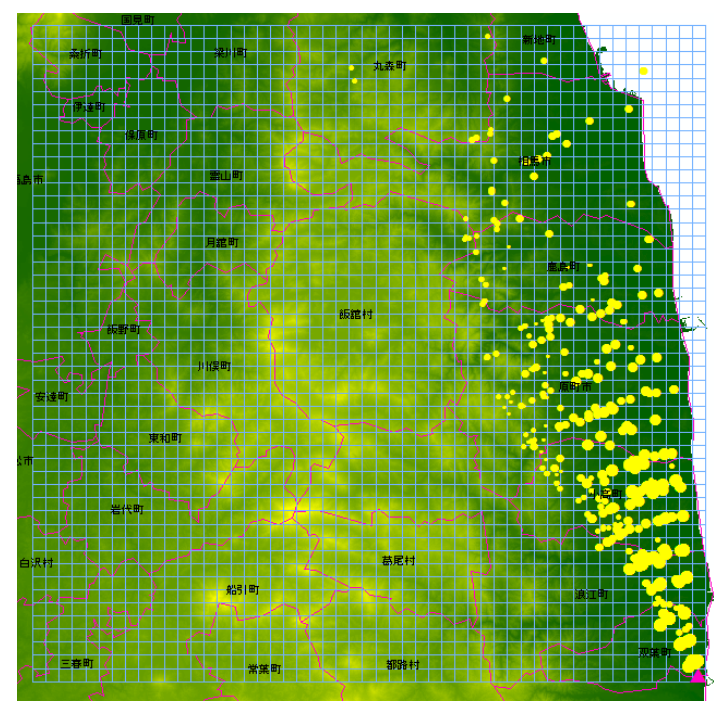

Figure 11. Particle trajectory estimated from wind directions and velocities on March 20

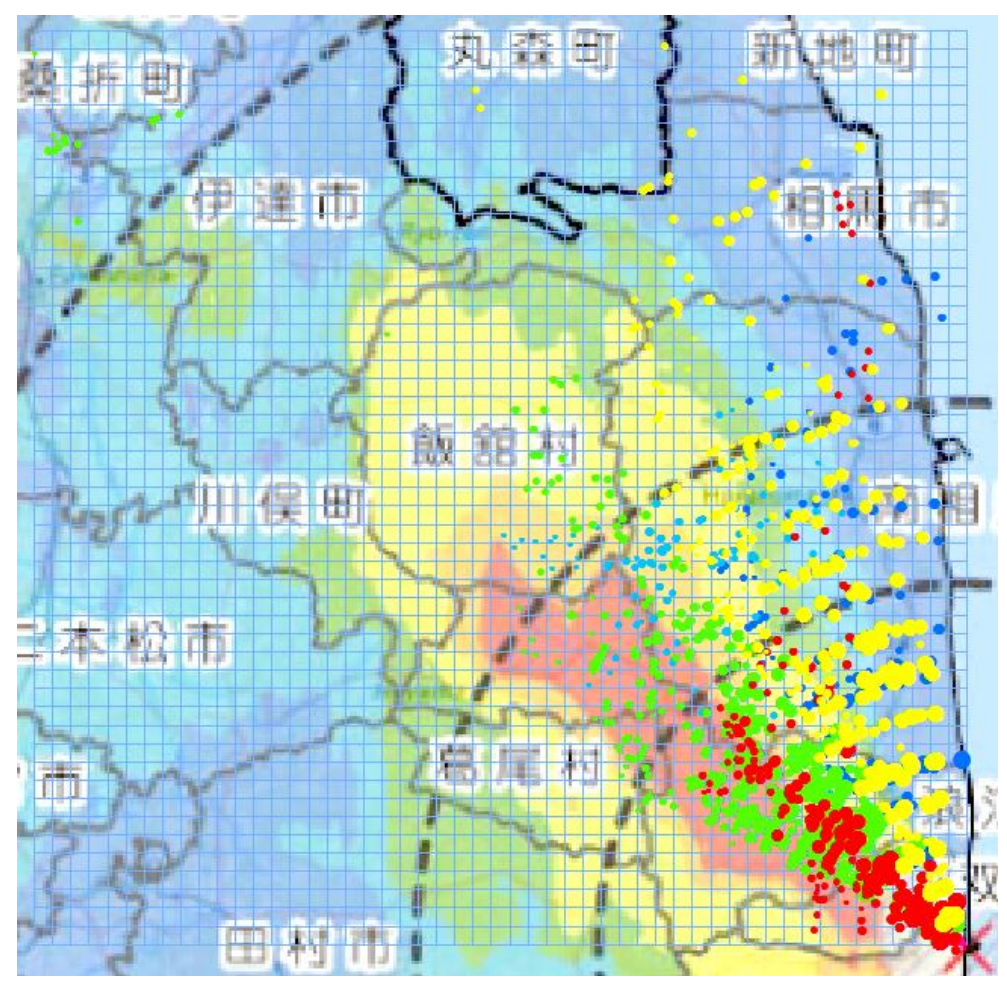

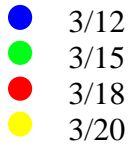

*Circles expressed particle sizes.

Figure 12. Radioisotope falling points on March 12, 15, 18, and 20

\subsection{Time series of spatial dose and precipitation}

The results of advection calculation for radioisotopes in this time are shown in Figure 13.

For these days precipitation was little and precipitation rates were 0.5 to $1.5 \mathrm{~mm} / \mathrm{h}$, therefore, pollution was wet deposit except March 15. Figure 13 shows a time series of spatial dose and dry- wet deposition ratios, wet by $6.5 \%$ and dry by $93.5 \%$, respectively.

That is, pollution on northwest of Fukushima Daiichi Nuclear Power Plant was contributed mainly by dry deposit from the leak of radioisotope such as vent. 


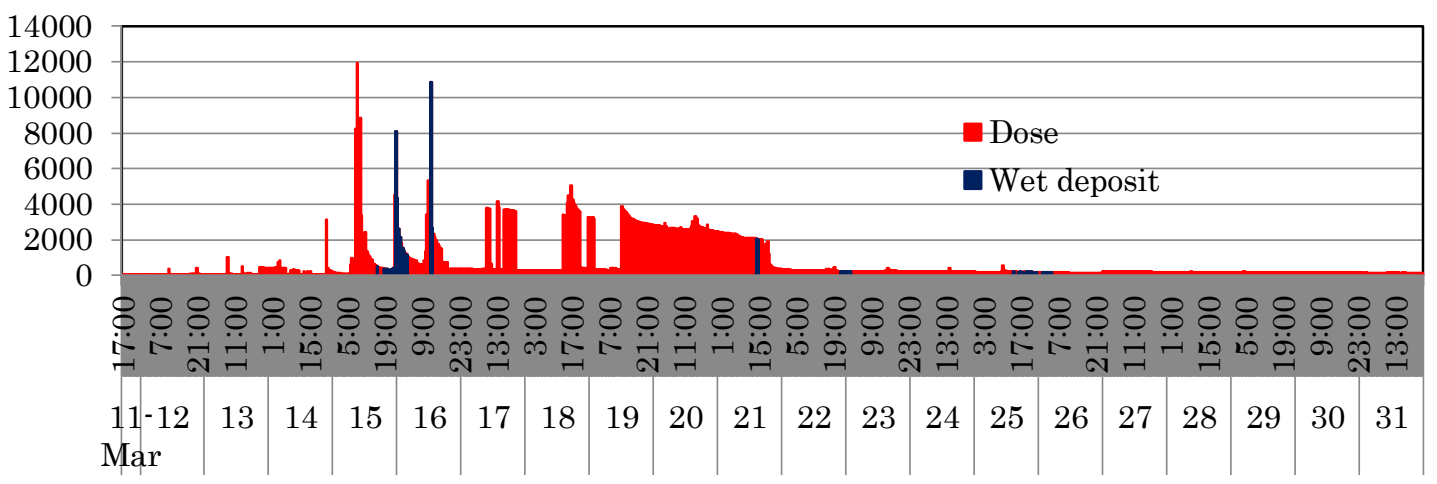

Figure 13. A time series of spatial dose (unit: $\mu \mathrm{Sv} / \mathrm{h}$ ) and the ratio of dry and wet deposits.

\section{DISCUSSION}

Pollution on northwest of Fukushima Daiichi Nuclear Power Plant started from hydrogen explosion on March 12, but the most of contribution for pollution was twice pollutions, on March 15 and 18. Pollution on March 12 was affected only by radioisotopes near $100 \mathrm{~m}$ height, but the result did not fit with aircraft monitoring results, and then this contribution was estimated to be small. On March 15, 18 and 20, radioisotopes under 200 m, 500 $\mathrm{m}$, and $500 \mathrm{~m}$ height flew into inland, respectively. Among them, on March 15 particles flied over the Abukuma Hills and it rained after flowing, thus, the result became intense pollution. On March 18 it was not rainy and wind was weak, and then pollution was limited for nearer areas on the northwest of the nuclear power plant. Therefore, the total pollution was contributed mostly by the radioisotope diffusion of hydrogen explosions and the leak of the containment vessel. Moreover, the pollution distribution was determined mainly by geography. On any days radioisotopes were blocked out by the Abukuma Hills, especially, against mountains with altitude 500 to $1192 \mathrm{~m}$ radioisotopes fell down on the slopes of and adsorbed in contaminant.

\section{CONCLUSIONS}

From above results, next conclusions were led.

(1) Advection calculation for radioisotopes using a particle model does not request any parameters, which an atmospheric diffusion model uses, atmospheric stability, the source height, a mixing layer height, and a deposition rate. Although a particle model is a simple method, the overall migration process for radioisotopes was estimated.

(2) Pollution on northwest of Fukushima Daiichi Nuclear Power Plant was mainly by emission on March 15, 18, and 20.

(3) Pollution of Fukushima Daiichi Nuclear Power Plant was limited because diffusion of radioisotopes was controlled by mountains with altitude $500 \mathrm{~m}$ to $1192 \mathrm{~m}$.

\section{FUTURE ASSIGNMENTS}

To improve fitness with monitoring results, difference of deposit each nucleus should be considered as future assignment. Moreover, for radioisotope migration after deposit, next geographic elements should be evaluated.

(1) Discharge from rivers and groundwater

(2) Absorption and runoff for land uses or land covers

(3) Effect by transportation: vehicles and railroads
By using approximation of these items in simple ways, emission by explosions and leaks, advection diffusion in the air, and migration on the earth surface should be estimated near future. Finally, instantaneous diffusion damage prediction in the accident and trial simulation for atmospheric diffusion before the accident should be solved with such simple ways.

\section{REFERENCES}

Japan Atomic Energy Agency, Atmospheric emission estimation for 131I and137Cs from Fukushima Daiichi Nuclear Power Plant accident, Part 2, 63rd Nuclear Safety Commission, 2011

Ohara, T. et al., Present state and problems on atmospheric transportation and depositions of radioisotope materials, Nuclear Science and Engineering Center, Open Workshop, 2012

Tokyo Electric Power Company, Atmospheric emission estimation for radioisotopes from Fukushima Daiichi Nuclear Power Plant accident, 2012

Meteorological Bureau, Forecast Department, Technical report on delivery information (meteorology): Hourly atmospheric analysis, GPV, Vol. 196, 2006.

Meteorological Bureau,

http://www.jma.go.jp/jma/kishou/know/kurashi/kaiseki.html

Ministry of Land, Infrastructure, Transport and Tourism, national land policy department,

http://nlftp.mlit.go.jp/ksj/gml/datalist/KsjTmplt-G04-a.html

Ministry of Education, Culture, Sports, Science and Technology, Aerial monitoring, 2011.

National Institute of Informatics, Meteorological data archive around Fukushima Daiichi Nuclear Power Plant.

http://agora.ex.nii.ac.jp/earthquake/201103eastjapan/weather/data/wind-20110311/surf/

Tokyo Electric Power Company, Monitoring state on Fukushima Daiichi Nuclear Power Plant.

http://www.tepco.co.jp/nu/fukushima-np/f1/indexold-j.html 